UCRL-JC-126856

PREPRINT

\title{
Evidence of Critical Scaling Behavior During Vapor Phase Synthesis of Continuous Filament Composites
}

\author{
J.H. Kinney \\ D.L. Haupt
}

This paper was prepared for submittal to the Materials Research Society 1996 Fall Meeting

Boston, MA

December 2-6, 1996

February 1997

This is a preprint of a paper intended for publication in a journal or proceedinga. Since changes may be made before publication, this preprint is made available with the understanding that it will not be cited or reproduced without the permisaion of the author. 


\section{DISCLAIMER}

This document was prepared as an accoum of work sponsored by an agency of the United States Government. Neither the United States Government nor the Uni versity of California nor any of their employees, makes any warranty, express or implied, or assumes any legal liability or responsibility for the accuracy, completeness, or usefulness of any information, apparatus, product, or process disclosed, or represents that its use woul d not infringe privately owned rights. Reference herein to any specific commercial products, process, or service by trade name, trademark, manufacturer, or otherwise, does not necessarily constitute or imply its endorsement, recommendation, or favoring by the United States Government or the University of Califomia. The views and opinions of authors expressed herein do not necessarily state or reflect those of the United States Government or the University of California, and shall not be used for advertising or product endorsement purposes. 


\title{
Evidence of critical scaling behavior during vapor phase synthesis of continuous filament composites
}

\author{
J.H. Kinney") and D. L. Haupt \\ Chemistry and Materials Science Deparıment, Lawrence Livermore National Laboratory. \\ Livermore, California 94550
}

(Received 1 August 1996; accepted 12 November 1996)

\begin{abstract}
We present experimental measurements of the accessible pore fraction in ceramic matrix composites during consolidation by vapor phase infiltration. For two topologically distinct filament architectures, the accessible pore fraction decreased during consolidation with a power law decay and a critical scaling exponent of $0.41\left(R^{2}=0.97\right)$. A three-

dimensional analysis of the percolating pores revealed that the structures became topologically equivalent and simply connected near the critical density.
\end{abstract}

Chemical Vapor Infiltration (CVI) is an important method for fabricating continuous filament composites. $^{12}$ In CVI, a reactive vapor phase is infiltrated at high temperature through woven reinforcing filaments. The growth of a matrix phase occurs from decomposition of this precursor, and continues for as long as the vapor can access the pores. Ideally, the pore consolidation should occur uniformly, so that access of the vapor is maintained until the composite is fully dense (i.c., the accessible pore fraction, $X^{\wedge}$, should remain close to unity). Though recent experimental data appear to suggest that pores close off uniformly, ${ }^{3}$ these data may be misleading, in that the method used to measure porosity (Archimedes' principle) could not distinguish between accessible porosity $\left(X^{\wedge}\right)$ and surface-connected porosity that didn't belong to the percolating cluster. In this study, we have used $x$-ray microtomography to directly determine $X^{\wedge}$ as a function of composite density during infiltration. Our observations are inconsistent with uniform deposition, and suggest instead that deposition is govemed by critical scaling behavior near final consolidation. The value of the critical scaling exponent measured in this study was 0.41 , identical to that predicted by three-dimensional bond and site percolation models.

Percolation theory has been applied to a number of physical phenomena. It has been particularly successful as a framework for describing fluid transport through porous media (see Ref. 4). According to percolation theory, pore consolidation during CVI should follow critical scaling of the form

$$
X^{A} \times\left(\rho-\rho_{i}\right)^{\beta},
$$

where $\rho$ is the density (dimensionless volume fraction) of pores, $\rho_{c}$ is a critical pore density below which the

\footnotetext{
${ }^{21}$ Address all comespondence to this author.
}

pore network no longer percolates, and $\beta=0.41$ is the universal topological exponent for a three-dimensional lattice (see Ref. 5). However, measuring $X^{\wedge}$ is a difficult experimental task. Though there have been studies that have attempted to characterize the microstructures of these materials, ${ }^{6}$ it has proved impossible to describe the connectivity of the pores with two-dimensional images.

Three-dimensional microtomography has been shown to be well suited for imaging porous structures. ${ }^{7-9}$ With parallel data acquisition, it is possible to image a $1 \mathrm{~cm}^{3}$ specimen in a few minutes. ${ }^{10.11}$ Reconstruction of the three-dimensional images can be done in a few hours on a computer workstation. Because of this, quantifying the evolving connectivity of the pore networks during vapor phase synthesis is no longer an impractical task. ${ }^{12}$

This study measured $X^{\wedge}$ as a function of total pore density during CVI. Woven Nicalon (amorphous SiC. Nippon Carbon) filament cloths (500 fibers per tow) were prepared with two topologically distinct square weave architectures: $0^{\circ} / 90^{\circ}$ and $0 \% / 45^{\circ}$ (where the nomenclature refers to the angular rotation between adjacent cloth layers). The specimens ranged in diameter from $6-8 \mathrm{~mm}$ and were approximately $8 \mathrm{~mm}$ in length. They were made by stacking multiple layers of Nicalon cloth into cylindrical graphite reaction chambers. The chambers were maintained at $975^{\circ} \mathrm{C}$ while a reactant gas of $\mathrm{H}_{2}$, bubbled through methyltrichlorosilane (MTS, a vapor precursor of $\beta-\mathrm{SiC}$ ), was forced through the graphite tubes and the cloth layups - a method known as isothermal forced flow CVI. The experimental procedure has been described in more detail elsewhere. ${ }^{8}$

The specimens were imaged prior to infiltration. and at different stages during consolidation, with threedimensional $\mathrm{x}$-ray microtomography using synchrotron radiation at the Stanford Synchrotron Radiation Laboratory. The three-dimensional images of the porosity were converted to binary data with the volume elements (voxels) being identified as belonging either to solid 
material or to void space. A modification of the cluster labeling method of Hoshen and Kopelman ${ }^{13}$ was used to identify the accessible (percolating) pores and all closed off pores that were no longer connected to the vapor source. ${ }^{14}$ This provided a means for exactly determining the fraction of the porosity $X^{\wedge}$ that remained connected to the vapor source.

The decrease in $X^{\wedge}$ as a function of specimen density for both architectures is shown in Fig. 1. Initially, all pores were interconnected, and the accessible pore fraction remained close to unity. At about $80 \%$ density, however, there was a pronounced decrease in the accessible pore fraction as pores closed off and became disconnected. The $\log$ of $X^{\wedge}$ for the $0^{\circ} / 45^{\bullet}$ weave is shown versus the $\log$ of $\left(\rho-\rho_{c}\right)$ in Fig. 2 with a least squares fit through the six data points nearest the critical density. The coarseness of the time resolution for the $0 \% / 90^{\circ}$ weave precluded a similar analysis. The decrease in $X^{\wedge}$ correlates well with a critical scaling exponent $\beta=0.41\left(R^{2}=0.97\right)$, and a critical pore density of $\rho_{c}=0.07$ (our best estimate of the critical density from the data). This is in excellent agreement with percolation theory for three-dimensional lattices, where a value of $\beta=0.41$ has been calculated. ${ }^{5}$ More specimens will be required to measure the statistical distribution of the data points and better determine the critical density.

The Euler characteristic, $\chi$, of a three-dimensional structure is related to the topological invariants:

$$
\chi=\beta_{0}-\beta_{1}+\beta_{2},
$$

where $\beta_{0}$ is the number of individual clusters, $\beta_{1}$ is the connectivity (number of handles), and $\beta_{2}$ is the number of enclosed surfaces or inclusions. For the percolating pore alone, $\beta_{0}=1$ and $\beta_{2}$ is identically zero. The

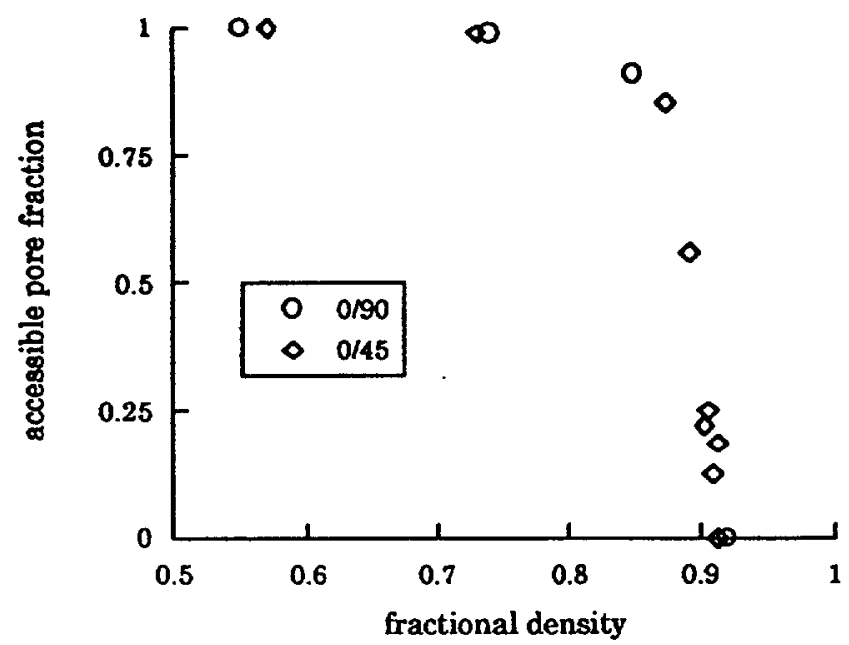

FIG. 1. The fraction of accessible porosity, $X^{A}$. as a function of composite density (fraction of theoretical). The open circles represent $0 \% / 90^{\circ}$ weave, whereas the open diamonds are the $0^{\circ} / 45^{\circ}$ weave. The rapid decrease in $X^{A}$ is characteristic of critical scaling behavior.

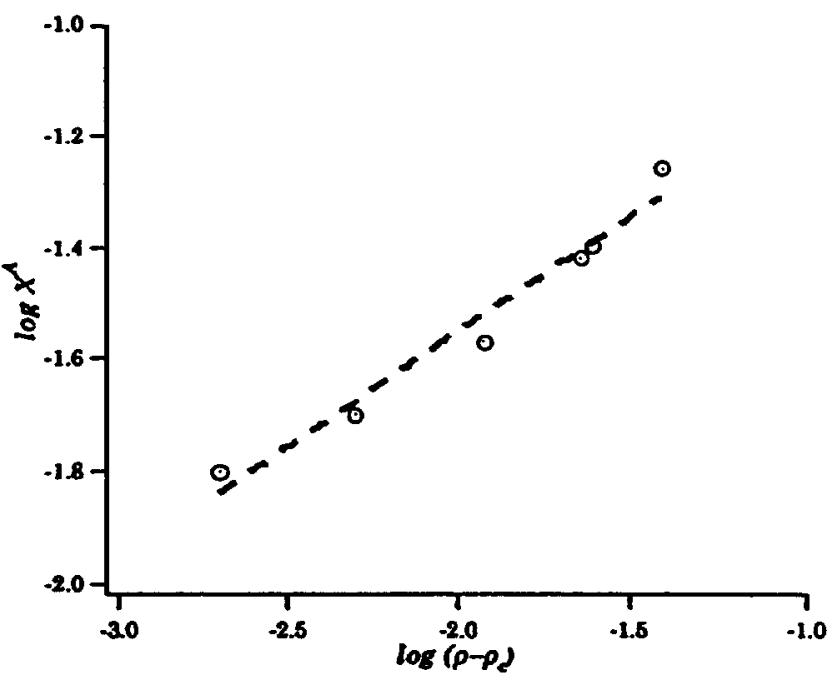

FIG. 2. A least squares fit to the $\log -\log$ plot of the six data points closest to the critical density for the $0 \% / 45^{\circ}$ weave. For a critical density of 0.07 . the critical scaling exponent is 0.41 . in excellent agreement with calculated values for three-dimensional lattices.

connectivity of the percolating pore is then determined by $\beta_{1}=1-\chi$. Using Feldkamp's algorithm for computing $\chi$, it is easy to calculate the connectivity for the percolating pore structures within the composite. ${ }^{15}$

The connectivity of the percolating pore is shown in Fig. 3. Initially, the $0 \% / 45^{\circ}$ architecture had approximately twice the connectivity of the $0 \% 190^{\circ}$ weave, as expected from geometric considerations. As the infiltration proceeded pore channels closed off, the connectivity decreased, and the percolating pore became simply connected $\left(\beta_{1}=0\right)$. For the $0^{\circ} / 90^{\circ}$, on the other hand, there was an initial increase in the connectivity because of the

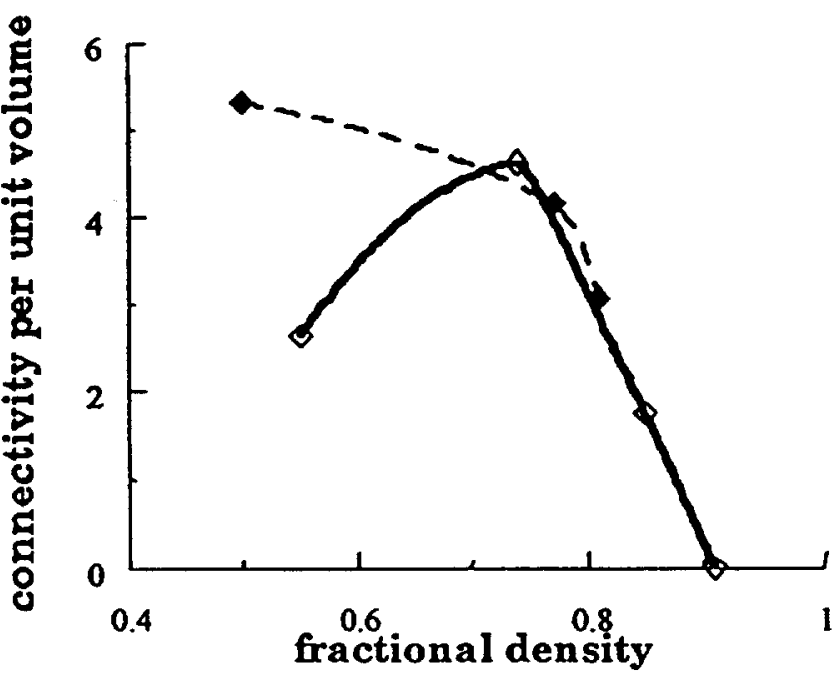

FIG. 3. The connectivity per cubic $\mathrm{mm}$ as measured for both the $0^{\circ} / 90^{\circ}$ (open diamonds) and $0 \% / 45^{\circ}$ (closed diamonds) weaves. Inilially quite different. the connectivity converges near $80 \%$ theoretical density. Near the critical density. the pore networks become simply connected. 
formation of matrix bridges between the cloth layers. At about $80 \%$ density the two architectures evolved into topologically equivalent structures, and remained equivalent until infiltration ceased.

We observed no difference in $\rho_{c}$ for the two architectures. The higher initial connectivity in the $0 \% 145^{\circ}$ weave implies a higher initial coordination number $Z$. Although theoretical and computational arguments suggest that $\rho_{c}$ should decrease in proportion to $1 / Z$ for $Z$ greater than three or four, ${ }^{16}$ in this study we found that the connectivity of the $0^{\circ} / 90^{\circ}$ weave increased during the initial stages of infiltration. If the coordination number increases during CVI, we may violate the expected asymptotic behavior. This could have a significant impact on attempts to decrease $\rho_{c}$ by controlling the initial fiber architecture, since a naive application of percolation theory would suggest that $\rho_{c}$ could be increased by increasing the initial connectivity of the fiber preform.

The agreement between our experimentally measured value of the critical scaling exponent and those from site and bond simulations on three-dimensional lattices is evidence that CVI is another class of problems that can be described by percolation theory. In addition, the trend toward formation of topologically equivalent pore networks. regardless of starting architecture, may be evidence that the topological exponents are universal in this system, and that $\rho_{c}$ may be insensitive to the fiber architecture.

The critical scaling behavior that we observed in this study has not been reproduced by traditional models of CVI. ${ }^{17}$ We believe that the models fail because they do not incorporate the random defects and irregularities in pore sizes that occur in physical systems, and because they assume that deposition is uniform throughout infiltration. Although deposition may be uniform in the early stages of infiltration, the closure of pore throats will ultimately dominate the infiltration process. The smallest of these throats will close first and, since they are randomly distributed, this closure will give the appearance of random bond breaking. Recently, Starr ${ }^{18}$ was able to simulate critical scaling behavior in CVI by carefully choosing a heterogeneous initial distribution of pore radii. We hypothesize that these heterogeneities in the initial pore structures are what give rise to the critical scaling and universality that we observed in CVI of continuous filament composites.

These results provide an easily measurable example of critical scaling behavior in a mesoscopic evolving porous structure. In addition, an understanding of the physical laws that govem vapor phase synthesis is vital for the development of new fiber architectures or filling strategies that may eventually decrease the cost and improve the mechanical quality of continuous fiber ceramic matrix composites. Future modeling of composite consolidation will have to incorporate realistic statistical descriptions of the heterogeneous distribution of porosity in the fiber preforms.

\section{ACKNOWLEDGMENTS}

Past support from the Advanced Industrial Materials Program, United States Department of Energy, is appreciated. The authors acknowledge fruitful discussions with T.M. Breunig (University of California, San Francisco), C. Henry (UC Davis), and A.J.C. Ladd (Lawrence Livermore National Laboratory). This work was performed under the auspices of the U.S. DOE W-7405-Eng-48 at LLNL.

\section{REFERENCES}

I. T. M. Besmann. B. W. Sheldon. R. A. Lowden. and D. P. Stinton. Science 253. 1104 (1991).

2. S. Vaidyaraman, W. J. Lackey. G. B. Freeman, P:K. Agrawal. and M.D. Langman, J. Maler. Res. 10.1469 (1995).

3. T.M. Besmann. J.C. McLaughlin, and H-T. Lin. J. Nucl. Mater. 219. 31 (1995).

4. M. Sahimi. Applications of Percolation Theory (Taylor and Francis. Bristol. PA. 1994).

5. D. Staufier and A. Aharony. Introduction to Percolation Theory. 2nd ed. (Taylor and Francis. London. 1992).

6. G. B. Freeman, T.L. Starr. and T.C. Elston, in Chemical Vapor Deposition of Refractory Metals and Ceramics, edited by T.M. Besmann and B. M. Gallois (Mater. Res. Soc. Symp. Proc. 168. Pitusturgh. PA, 1990). p. 49.

7. P. Spanne. J.F. Thoven. C. J. Jacquin. W. B. Lindquist, et al. Phys. Rev. Lelt. 73. 2001 (1994).

8. J.H. Kinney, T. M. Breunig. T.L. Starr. D. Haupt. a al.. Science 260, 789 (1993).

9. B.P. Flannery, H.W. Deckman. W.G. Roberge. and K.L. D'Amico. Science 237, 1439 (1987).

10. U. Bonse el al., Nucl. Instrum. Methods. Phys. Res. A 246, 43 (1986).

11. J. H. Kinney and M.C. Nichols. Annu. Rev. Maler. Sci. 22, 121 (1992).

12. J. H. Kinney. C.P. Henn'. D. L. Haupt, and T.L. Starr, Appl. Composite Mater. 1. 325 (1994).

13. J. Hoshen and R. Kopelman. Phys. Rev. B 14, 3438 (1976).

14: J.H. Kinney. D. L. Haupi, M.C. Nichols. T. M. Breunig, et al.. Nucl. Instrum. Methods. Phys. Res. A 347, 480 (1994).

15. L.A. Feldkamp, S. A. Goldstein. A.M. Parfilt, et al., J. Bone Miner. Res. 4. 3 (1989).

16. C. Kurrer and K. Schulten. Phys. Rev. E 48, 614 (1993).

17. B. W. Sheldon and T. M. Besmann. J. Am. Ceram. Soc. 74, 3046 (1991).

18. T. L. Starr. J. Mater. Res. 10. 2360 (1995). 


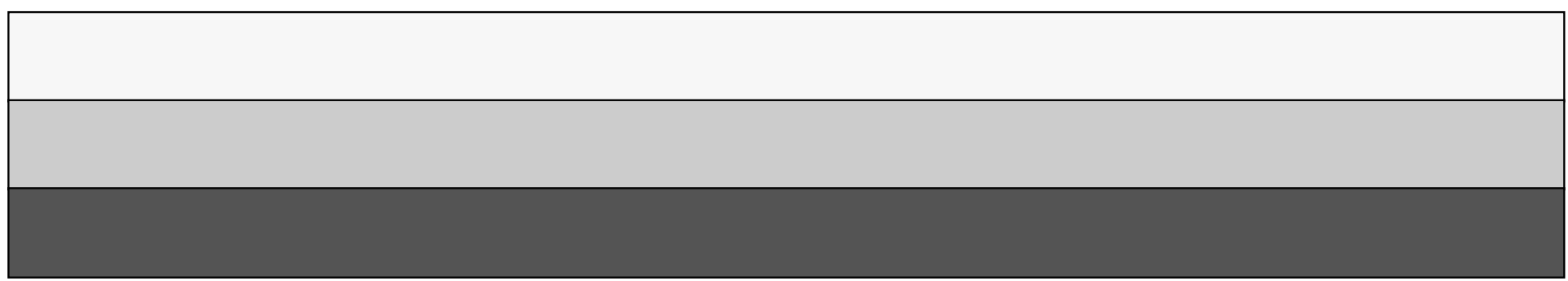

